\title{
Improved method of reactive loading for miniaturisation of transmission lines with minimal degradation in performance
}

ISSN 1751-8725

Received on 12th August 2014 Accepted on 17th December 2014 doi: 10.1049/iet-map.2014.0547 www.ietdl.org

\author{
Taejun Lim ${ }^{1}$, Seungku Lee ${ }^{2}$, Yongshik Lee ${ }^{1 凶}$ \\ ${ }^{1}$ Department of Electrical and Electronic Engineering, Yonsei University, Seoul 120-749, Korea \\ ${ }^{2}$ Department of Electrical Engineering and Computer Science, University of Michigan, Ann Arbor, MI 48109, USA \\ 凶-mail: yongshik.lee@yonsei.ac.kr
}

\begin{abstract}
This study presents an improved method of constant reactive loading for the miniaturisation of quarterwavelength transmission lines. The method provides an optimum location for loading, which maintains the electrical characteristics before and after miniaturisation. Therefore circuits based on transmission lines can be miniaturised with negligible deterioration in performance. This simple yet highly effective method is especially attractive for popular multi-section circuits, in which a number of sections are cascaded for wider bandwidths. Experimental results of various Chebyshev multi-section impedance transformers not only verifies the effectiveness of this method in bandwidths as large as $99 \%$ with a size reduction of more than $55 \%$, but also demonstrates the method's flexibility of design.
\end{abstract}

\section{Introduction}

Quarter-wavelength transmission lines are essential components for various microwave circuits including branch-line hybrid couplers, ring hybrid couplers, Wilkinson power dividers, filters and impedance transformers [1]. Recently, progress has been made in miniaturising a quarter-wavelength transmission-line section and/or circuits based on such lines. Among them, the methods of reactive loading [2-5] have been regarded as the most attractive method because of their simplicity. These methods have been widely applied in the miniaturisation of various planar circuits including Wilkinson power dividers [6] and bandpass filters [7]. However, the methods rely on the equivalence of the electrical performance before and after miniaturisation only at the design frequency. Therefore performance deterioration is inevitable at other frequencies.

Similar methods such as periodic loading [8-10], artificial transmission lines [11-16] and distributive loading [17-19] have demonstrated considerable size reduction for branch-line hybrid couplers [11-19], ring hybrid couplers [10-12], Wilkinson power dividers [8] and dual-band filters [9]. However, the major disadvantages of these methods include less accuracy when the number of loading is small, and there is unwanted coupling between adjacent lines. These issues need to be addressed through intensive full-wave simulations.

In addition, structural modification has been a popular miniaturisation method. For example, the integration of the composite right-/left-handed technique with a fractal structure [2023] demonstrated not only a size reduction factor of up to $90 \%$ [21], but also a substantial bandwidth enhancement for a power divider [22] or a balun [23]. Other methods include a defected ground plane, which allows for harmonic suppression [24-26] or exhibits low loss [27-29]; a complementary-conducting-strip line [30]; a perforated ground metal [31]; and lattice-ladder topology [32]. The major drawbacks are high fabrication cost and a relatively complex design procedure that relies heavily on full-wave simulation.

This paper presents an improved reactive loading method for the miniaturisation of transmission lines that provide minimal performance degradation. Compared with previous state-of-the-art methods of miniaturisation [11-32], the proposed method is based on popular reactive loading. The structural simplicity of the method allows for accurate estimation of the performance with circuit simulations, which expedites the design process. In this paper, an optimal location of reactive loading is determined; this location retains the properties of a quarter-wavelength transmission line before and after loading. Therefore, compared with previous miniaturisation methods based on reactive loading [2-10], a transmission-line circuit can be miniaturised with negligible degradation in performance. Furthermore, the proposed method is far more attractive, especially for multi-section circuits (in which a number of sections are cascaded to achieve wider bandwidths), since the performance of previous methods degrades even more at frequencies farther away from the design frequency.

For experimental verification, various multi-section Chebyshev impedance transformers are demonstrated. Minimal degradation in performance is observed in bandwidths as wide as $99 \%$ with a miniaturisation rate of more than $55 \%$.

\section{Miniaturisation of transmission line}

Fig. 1 shows the schematics of conventional quarter-wavelength and proposed transmission lines. These are loaded symmetrically with $n$ identical series inductors or shunt capacitors for miniaturisation, where $n$ is the total number of reactive elements. All transmission line sections in the miniaturised lines have the same characteristic impedance $Z_{L}$ or $Z_{C}$ for shunt-capacitive loading. It is assumed that all transmission line sections between two reactive elements are of a commensurate length $\theta_{2}$, whereas the length of the two end sections is $\theta_{1}$, which generally is different from $\theta_{2}$. Miniaturisation is achieved when the total electrical length $\theta=2 \theta_{1}$ $+(n-1) \theta_{2}$ is shorter than $90^{\circ}$.

Conditions of miniaturisation can be obtained with the $\boldsymbol{A B C D}$ parameters of the two lines

$$
\begin{gathered}
\boldsymbol{A} \boldsymbol{B} \boldsymbol{C} \boldsymbol{D}^{C}=\left[\begin{array}{cc}
0 & \mathrm{j} Z_{0} \\
\mathrm{j} Y_{0} & 0
\end{array}\right] \\
\boldsymbol{A} \boldsymbol{B} \boldsymbol{C} \boldsymbol{D}^{R}=\left[\begin{array}{ll}
a^{\mathrm{LH}} & b^{\mathrm{LH}} \\
c^{\mathrm{LH}} & d^{\mathrm{LH}}
\end{array}\right]\left[\begin{array}{ll}
a^{\mathrm{RH}} & b^{\mathrm{RH}} \\
c^{\mathrm{RH}} & d^{\mathrm{RH}}
\end{array}\right]
\end{gathered}
$$

where the superscripts $C$ and $R$ denote conventional 

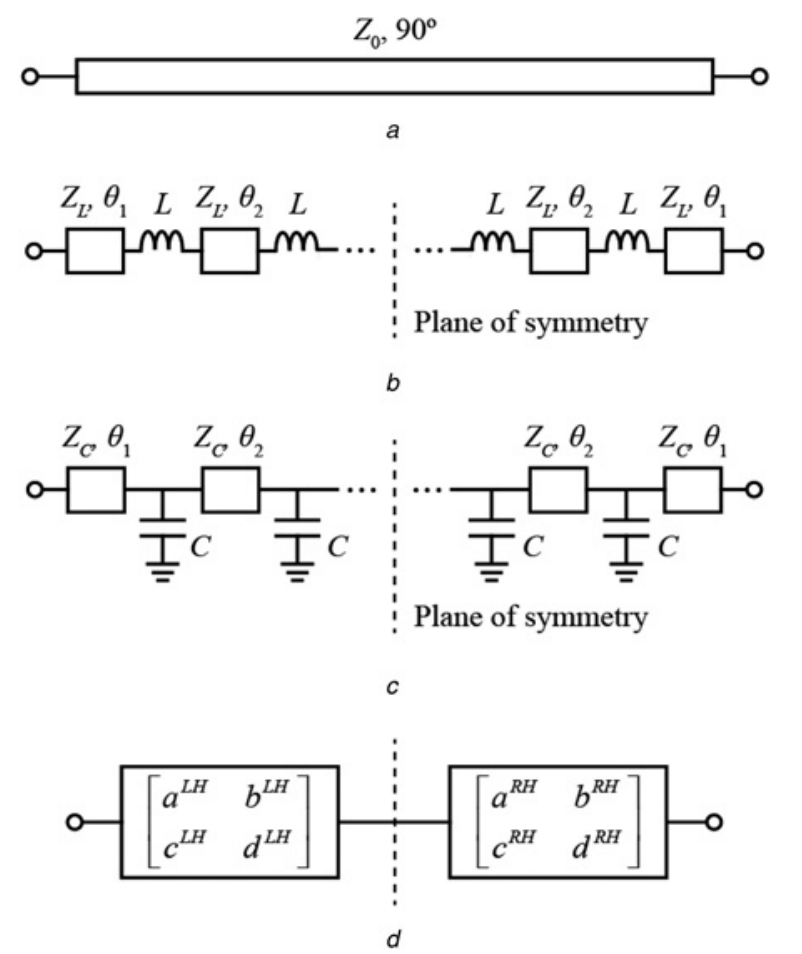

Fig. 1 Transmission lines

$a$ Conventional quarter-wavelength transmission line

$b$ Series-inductively loaded transmission line

$c$ Shunt-capacitively loaded transmission line

d $\boldsymbol{A B C D}$ matrix representation of reactively loaded lines

quarter-wavelength and reactively loaded transmission lines, respectively. The superscripts $\mathrm{LH}$ and $\mathrm{RH}$ denote the left-handed and right-handed parts of the miniaturised line, respectively.

Since the structure is symmetric with respect to its centre, the $\boldsymbol{A B C D}$ matrices of left and right parts are inverse to each other. In addition, $a^{\mathrm{LH}} d^{\mathrm{LH}}-b^{\mathrm{LH}} c^{\mathrm{LH}}=1$ and $a^{\mathrm{RH}} d^{\mathrm{RH}}-b^{\mathrm{RH}} c^{\mathrm{RH}}=1$, since the line is reciprocal. Therefore the two $\boldsymbol{A B C D}$ matrices are related as follows

$$
\left[\begin{array}{ll}
a^{\mathrm{RH}} & b^{\mathrm{RH}} \\
c^{\mathrm{RH}} & d^{\mathrm{RH}}
\end{array}\right]=\left[\begin{array}{ll}
d^{\mathrm{LH}} & b^{\mathrm{LH}} \\
c^{\mathrm{LH}} & a^{\mathrm{LH}}
\end{array}\right]
$$

Now, (1b) can be expressed as

$$
\boldsymbol{A} \boldsymbol{B} \boldsymbol{C} \boldsymbol{D}^{R}=\left[\begin{array}{cc}
a^{\mathrm{LH}} d^{\mathrm{LH}}+b^{\mathrm{LH}} c^{\mathrm{LH}} & 2 a^{\mathrm{LH}} b^{\mathrm{LH}} \\
2 c^{\mathrm{LH}} d^{\mathrm{LH}} & a^{\mathrm{LH}} d^{\mathrm{LH}}+b^{\mathrm{LH}} c^{\mathrm{LH}}
\end{array}\right]
$$

Finally, the design parameters of the miniaturised line can be obtained by equating the $\boldsymbol{A B C D}$ parameters of the conventional line in (1a) and the reactively loaded line in (3).

\section{$2.1 n=2$ case}

For $n=2$, the characteristic impedance $Z_{L}$ and the loading inductance $L$ for series-inductive loading in Fig. $1 b$ are

$$
\begin{gathered}
Z_{L}=Z_{0} \frac{2 \sin ^{2} \theta_{1}}{\sqrt{\sin ^{2}\left(2 \theta_{1}\right)+\sin ^{2} \theta_{2}}-\sin \theta_{2}} \\
L=Z_{L} \frac{\sin \left(2 \theta_{1}+\theta_{1}\right)-\sqrt{\sin ^{2}\left(2 \theta_{1}\right)+\sin ^{2} \theta_{2}}}{\omega_{0}\left[\sin ^{2}\left(\theta_{1}+\theta_{2} / 2\right)-\sin ^{2}\left(\theta_{1}-\theta_{2} / 2\right)\right]}
\end{gathered}
$$

and the characteristic impedance $Z_{C}$ and the loading capacitance $C$ for shunt-capacitive loading in Fig. $1 c$ are

$$
\begin{gathered}
Z_{C}=Z_{0} \frac{2\left(1-\sin ^{2} \theta_{1}\right)}{\sqrt{\sin ^{2}\left(2 \theta_{1}\right)+\sin ^{2} \theta_{2}}+\sin \theta_{2}} \\
C=\frac{1}{Z_{C}} \frac{\sin \left(2 \theta_{1}+\theta_{2}\right)-\sqrt{\sin ^{2}\left(2 \theta_{1}\right)+\sin ^{2} \theta_{2}}}{\omega_{0}\left[\sin ^{2}\left(\theta_{1}+\theta_{2} / 2\right)-\sin ^{2}\left(\theta_{1}-\theta_{2} / 2\right)\right]}
\end{gathered}
$$

In (4) and (5), $\omega_{0}=2 \pi f_{0}$ is the angular velocity at the design frequency where the electrical length of the lines before and after miniaturisation is $90^{\circ}$.

The $n=2$ case is the generalised version of previous methods of reactive loading since, for example, $\theta_{1}=0^{\circ}$ corresponds to the case of reactive loading at the two ends as in $[2,4]$, and $\theta_{2}=0^{\circ}$ corresponds to that of centre loading [5]. For the former, both the numerator and the denominator in (4b) and (5b) vanish. In this case, the conditions must be derived from (1) and (3) with $\theta_{1}=0^{\circ}$. The conditions are

$$
\begin{gathered}
Z_{L}=Z_{0} \sin \theta_{2} \\
L=\frac{Z_{L}}{\omega_{0}} \cos \theta_{2}
\end{gathered}
$$

for series-inductive loading, and

$$
\begin{gathered}
Z_{C}=Z_{0} \csc \theta_{2} \\
C=\frac{1}{\omega_{0} Z_{\mathrm{C}}} \cos \theta_{2}
\end{gathered}
$$

for shunt-capacitive loading. When $\theta_{2}=2 \theta_{1}$, the method becomes equally spaced or periodic loading, as in [8]. The conditions are

$$
\begin{gathered}
Z_{L}=Z_{0} \frac{2 \sin ^{2} \theta_{1}}{(\sqrt{2}-1) \sin \left(2 \theta_{1}\right)} \\
L=Z_{L} \frac{\sin \left(4 \theta_{1}\right)-\sqrt{2} \sin \left(2 \theta_{1}\right)}{\omega_{0} \sin ^{2}\left(2 \theta_{1}\right)}
\end{gathered}
$$

for series-inductive loading, and

$$
\begin{gathered}
Z_{C}=Z_{0} \frac{2\left(1-\sin ^{2} \theta_{1}\right)}{(\sqrt{2}+1) \sin \left(2 \theta_{1}\right)} \\
C=\frac{1}{Z_{C}} \frac{\sin \left(4 \theta_{1}\right)-\sqrt{2} \sin \left(2 \theta_{1}\right)}{\omega_{0} \sin ^{2}\left(2 \theta_{1}\right)}
\end{gathered}
$$

for shunt-capacitive loading. These conditions for uniform loading are somewhat different from those in [8]. Those in (8)-(9) are based solely on the equality of $\boldsymbol{A B C D}$ parameters at the design frequency. Therefore the same performance is guaranteed before and after miniaturisation at the frequency. On the other hand, conditions in [8] are based on the transmission line theory, assuming that the loading reactance or susceptance is distributed uniformly along the line. Therefore a relatively large degradation of performance can be observed when $n$ is small.

\section{$2.2 n \geq 3$ case}

For $n=3$, the equations for the impedances $Z_{L}$ and $Z_{C}$ both become cubic functions. For series-inductive loading, the characteristic impedance $Z_{L}$ can be obtained by solving the following equations

$$
X_{1} Z_{L}^{3}+X_{2} Z_{L}^{2}+X_{3} Z_{L}+X_{4}=0
$$


where

$$
\begin{aligned}
X_{1}= & \cos ^{3} \theta_{1} \\
X_{2}= & Z_{0}\left\{-\cos ^{3} \theta_{1} \cos \theta_{2} \sin \theta_{2}-\cos ^{2} \theta_{1} \sin \theta_{1}\left(1+\sin ^{2} \theta_{2}\right)\right. \\
& \left.-\cos \theta_{1} \cos \theta_{2} \sin ^{2} \theta_{1} \sin \theta_{2}-\sin ^{3} \theta_{1} \sin ^{2} \theta_{2}\right\} \\
X_{3}= & Z_{0}^{2}\left\{-\cos ^{3} \theta_{1} \sin ^{2} \theta_{2}+\cos ^{2} \theta_{1} \cos \theta_{2} \sin \theta_{1} \sin \theta_{2}\right. \\
& \left.-\cos \theta_{1} \sin ^{2} \theta_{1}\left(1+\sin ^{2} \theta_{2}\right)+\cos \theta_{2} \sin ^{3} \theta_{1} \sin \theta_{2}\right\} \\
X_{4}= & Z_{0}^{3} \sin ^{3} \theta_{1}
\end{aligned}
$$

Using the solution of (10), the loading inductance $L$ can be obtained by the following equation

$$
L=Z_{L} \frac{Z_{L} \cos \left(\theta_{1}+\theta_{2}\right)-Z_{0} \sin \left(\theta_{1}+\theta_{2}\right)}{\omega_{0} \sin \theta_{2}\left(Z_{L} \cos \theta_{1}-Z_{0} \sin \theta_{1}\right)}
$$

In addition, for shunt-capacitive loading, the characteristic impedance $Z_{\mathrm{C}}$ can be found by solving the cubic equation

$$
Y_{1} Z_{C}^{3}+Y_{2} Z_{C}^{2}+Y_{3} Z_{C}+Y_{4}=0
$$

where

$$
\begin{aligned}
Y_{1}= & \sin ^{3} \theta_{1} \\
Y_{2}= & Z_{0}\left\{-\cos ^{3} \theta_{1} \sin ^{2} \theta_{2}+\cos ^{2} \theta_{1} \cos \theta_{2} \sin \theta_{1} \sin \theta_{2}\right. \\
& \left.-\cos \theta_{1} \sin ^{2} \theta_{1}\left(1+\sin ^{2} \theta_{2}\right)+\cos \theta_{2} \sin ^{3} \theta_{1} \sin \theta_{2}\right\} \\
Y_{3}= & Z_{0}^{2}\left\{-\cos ^{3} \theta_{1} \cos \theta_{2} \sin \theta_{2}-\cos ^{2} \theta_{1} \sin \theta_{1}\left(1+\sin ^{2} \theta_{2}\right)\right. \\
& \left.-\cos \theta_{1} \cos \theta_{2} \sin ^{2} \theta_{1} \sin \theta_{2}-\sin ^{3} \theta_{1} \sin ^{2} \theta_{2}\right\} \\
Y_{4}= & Z_{0}^{3} \cos ^{3} \theta_{1}
\end{aligned}
$$

Then, using the solution of (12), the loading capacitance $C$ can be obtained by the following equation

$$
C=\frac{1}{Z_{C}} \frac{Z_{0} \cos \left(\theta_{1}+\theta_{2}\right)-Z_{C} \sin \left(\theta_{1}+\theta_{2}\right)}{\omega_{0} \sin \theta_{2}\left(Z_{0} \cos \theta_{1}-Z_{C} \sin \theta_{1}\right)}
$$

As $n$ increases, the degree of the equation for $Z_{L}$ or $Z_{C}$ increases by the same amount. Thus, the equations become relatively complex to solve analytically for $n \geq 4$. In this paper, a generic numerical code has been written to obtain the design parameters that equalise the $\boldsymbol{A B C D}$ parameters in (3) with (1a) for an arbitrary $n$.

\section{Optimal location of reactive loading for minimal performance deterioration}

The miniaturisation method in the previous section relies only on the equivalence of $\boldsymbol{A B C D}$ parameters before and after loading, only at the design frequency. Therefore when a circuit is developed with such lines, performance deterioration at other frequencies is inevitable, causing bandwidth shrinkage [2]. In addition, properties such as passband response in cascaded circuits and isolation in multi-port circuits may deteriorate.

As seen in Section 2, the design parameters of a miniaturised transmission line are functions of the locations of reactive loading, that is, $\theta_{1}$ and $\theta_{2}$. This indicates that even if $\theta_{1}$ and $\theta_{2}$ are miniaturised to the same length $\theta$, performance degradation is different for a different set of $\theta_{1}$ and $\theta_{2}$. This suggests that an optimum location of loading may exist that minimises the performance deterioration because of miniaturisation.

For an ideal transmission line, comparing $S_{11}$ before and after miniaturisation is sufficient to assess degradation in performance because of miniaturisation. An example is shown in Figs. $2 a$ and $b$ for series inductively loaded lines of $\theta=2 \theta_{1}+\theta_{2}=30^{\circ}$ and $60^{\circ}$ with $n=2$. For each length, three different locations of reactive loading $(P=15,72$ and $90 \%)$ are considered, where

$$
P=\frac{\theta_{2}}{\theta_{1}+\theta_{2}} \times 100(\%)
$$

The location of loading $P=100 \%$ corresponds to reactive loading at the two ends, whereas $P=0 \%$ corresponds to reactive loading at the centre of the line; when $P=72 \%, 18 \theta_{1}=7 \theta_{2}$. For example, $P=72 \%$ when $\theta_{1}=13.125^{\circ}$ and $\theta_{2}=33.75^{\circ}$ for $\theta=60^{\circ}$. The design parameters can be calculated from (4) using the corresponding $\theta_{1}$ and $\theta_{2}$.

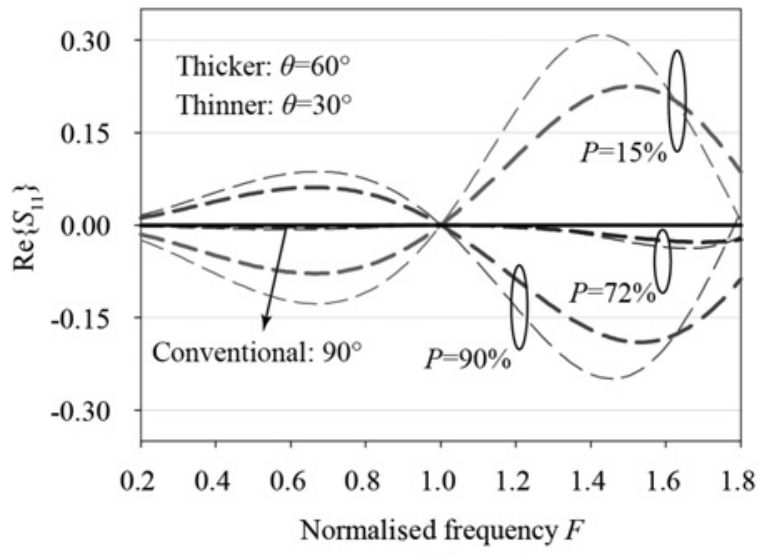

a

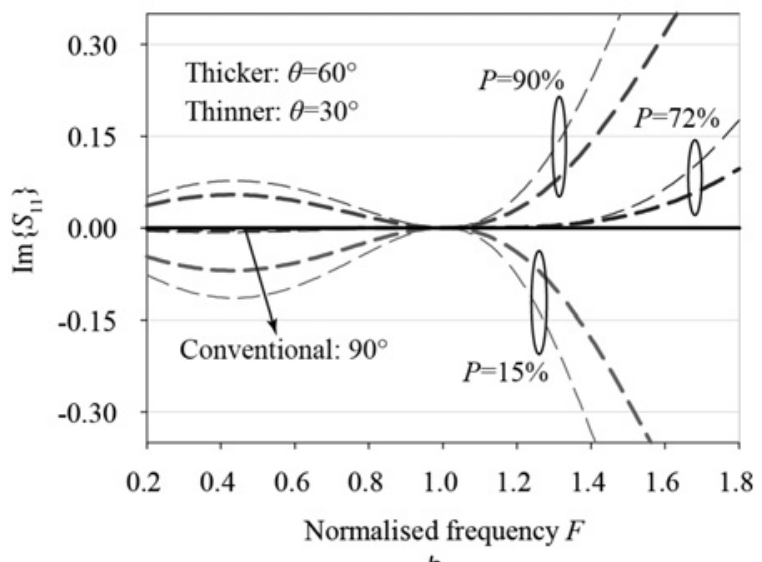

$b$

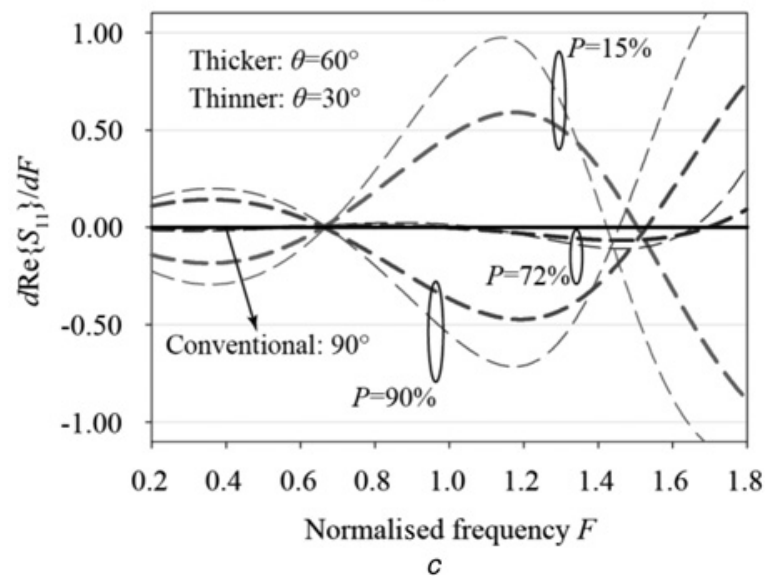

Fig. 2 Comparison of $S_{11}$ : ideal $\left(90^{\circ}\right)$ with arbitrary characteristic impedance and miniaturised with two $(n=2)$ series inductors at $P=15,72$ and $90 \%$

$a \operatorname{Re}\left\{S_{11}\right\}$

$b \operatorname{Im}\left\{S_{11}\right\}$

$c \mathrm{dRe}\left\{S_{11}\right\} / \mathrm{d} F$ 
Figs. $2 a$ and $b$ show that $\operatorname{Re}\left\{S_{11}\right\}=0$ and $\operatorname{Im}\left\{S_{11}\right\}=0$ at the centre frequency not only for the conventional, but also for the miniaturised lines, regardless of the values of $P$ or the miniaturised length $\theta$. However, although the ideal quarter-wavelength line remains perfectly matched at all frequencies, this is not the case for miniaturised lines, as evidenced by the changes in $S_{11}$ in Figs. $2 a$ and $b$. In addition, depending on $P$, degradation in matching may be substantial. It becomes obvious that a circuit based on such miniaturised transmission lines will experience a deterioration in performance. This suggests that, in addition to the equivalence of $\boldsymbol{A B C D}$ parameters (and therefore $Z, Y$ and $S$ parameters) at the design frequency, the miniaturisation method requires an additional condition to minimise degradation in performance.

The mathematical similarity of $S_{11}$ before and after miniaturisation is a direct measure of physical resemblance between the two lossless lines. Mathematically, two functions are equal when they intersect and have the same differential functions. Since $S_{11}=0$ for both lines, the derivatives of $S_{11}$ of both lines remain to be examined.

Before miniaturisation, the derivatives of both the real and imaginary parts of $S_{11}$ vanish at all frequencies. However, this is not the case for miniaturised lines, as shown in Fig. $2 c$, where the derivative of the real part is shown only because the derivative of the imaginary part is negligible. For example, the change in the derivatives of $S_{11}$ with respect to $F$ (frequency normalised by the design frequency) is significant for $P=15 \%$. However, the change becomes insignificant when $P=72 \%$, which indicates a mathematical similarity between the responses of ideal and miniaturised lines over a wide bandwidth. Since $\mathrm{d} \operatorname{Re}\left\{S_{11}\right\} / \mathrm{d} F=0$ at the design frequency $F=1$ for an ideal line, the combination of $\theta_{1}$ and $\theta_{2}$ that minimises $\left|\operatorname{dRe}\left\{S_{11}\right\} / \mathrm{d} F\right|$ at $F=1$ after loading is the optimum location of loading $\theta_{1}$ and $\theta_{2}$ or $P$ that minimises deterioration in performance because of loading.
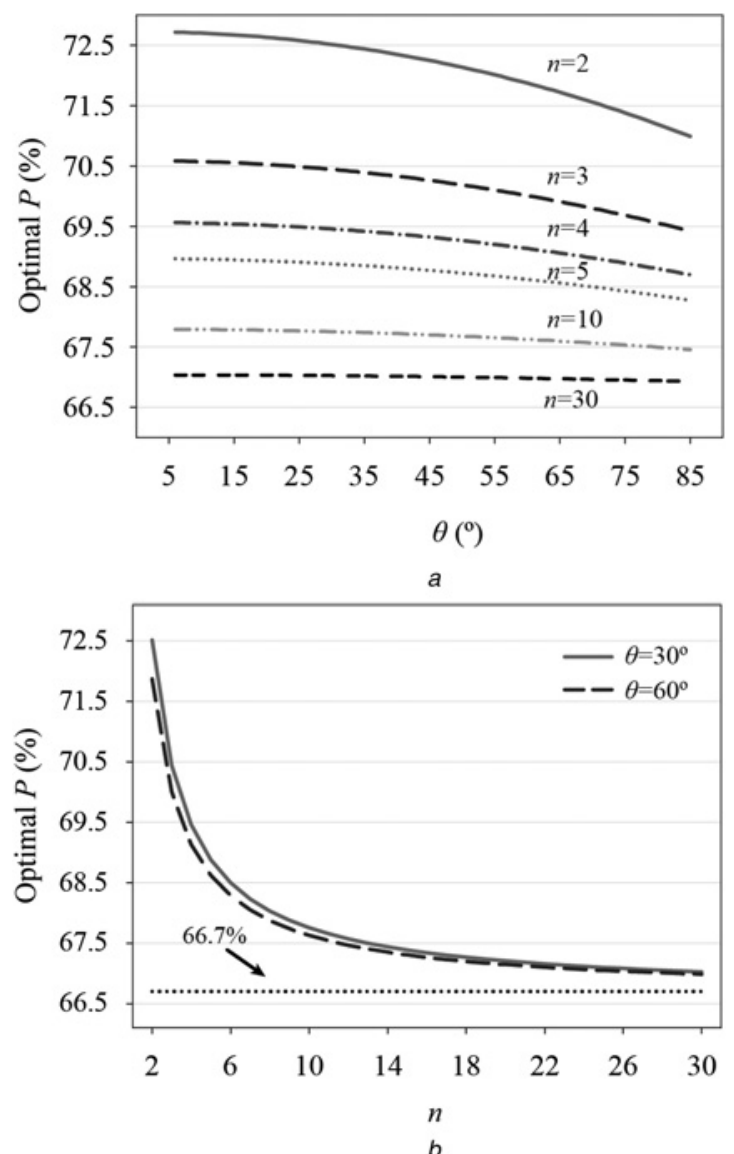

Fig. 3 Optimal location of reactive loading with respect to design variables $a$ Miniaturisation length $\theta$

$b$ Number of loading $n$
Fig. 3 shows the calculated optimal $P$. Since the series-inductive loading and shunt-capacitive loading methods are dual structures, the optimal locations for both methods are identical. Although the optimum location remains practically the same with respect to the length of miniaturisation $\theta$, a minor difference is observed with respect to $n$. For example, $P=72 \%$ can be considered the optimum location for $n=2$ regardless of $\theta$. The optimum location decreases as $n$ increases, eventually converging to $66.7 \%$, which corresponds to equally spaced loading. This also validates the proposed analysis, since for an infinitely large $n$, both $\theta_{1}$ and $\theta_{2}$ become sufficiently infinitesimal that the loading capacitance (or inductance) can be treated as a distributed parameter of the transmission line section, which then makes the line effectively synthetic.

The importance of the optimum location of loading can be verified in Fig. 4, which compares the calculated effective characteristic impedance $Z_{\text {eff }}$ of quarter-wavelength miniaturised lines with $n=2$ at various loading locations. $Z_{\text {eff }}$ is the effective characteristic impedance of a virtual synthetic transmission line that has the same electrical characteristics as the reactive-loaded line. $Z_{\text {eff }}$ is the same as the characteristic impedance $Z_{0}$ of its conventional counterpart at the design frequency. However, because of the frequency-dependent characteristics of the miniaturised line, $Z_{\text {eff }}$ changes as the frequency changes. Compared with other locations, the bandwidth in which $Z_{\text {eff }}$ remains $50 \Omega$ is remarkably larger for $P=72 \%$, regardless of $\theta$. Although it is not shown here, $P=72 \%$ also provides the widest bandwidth over which the effective electrical length is maintained at $90^{\circ}$. This shows that the optimal location in Fig. 3 is indeed the location that maintains the electrical properties of a transmission line before and after miniaturisation. Finally, because of the low-pass-like structure of the proposed method of reactive loading, performance degradation in miniaturised lines is more severe at the higher band edge than at the lower edge.

Fig. 5 shows examples of design curves. For a higher degree of miniaturisation, the impedance level decreases for inductive loading, whereas it increases for capacitive loading. Therefore, regarding the impedance levels, the former is more suitable for miniaturisation of high-impedance lines, and the latter is more suitable for low-impedance lines. Fig. 5 also shows that the characteristic impedance of transmission line sections in a miniaturised line remains practically the same regardless of $n$. A higher degree of miniaturisation is achieved with more reactive elements and/or higher reactance. However, for the same $\theta$, the total loading reactance is maintained at nearly the same value regardless of $n$. This, together with the fact that performance degradation such as bandwidth shrinkage is less severe for a higher $n$ (i.e. when the line is loaded more densely), is a clear indication that the optimal location is the location that distributes the loading capacitance (or inductance) the most uniformly along

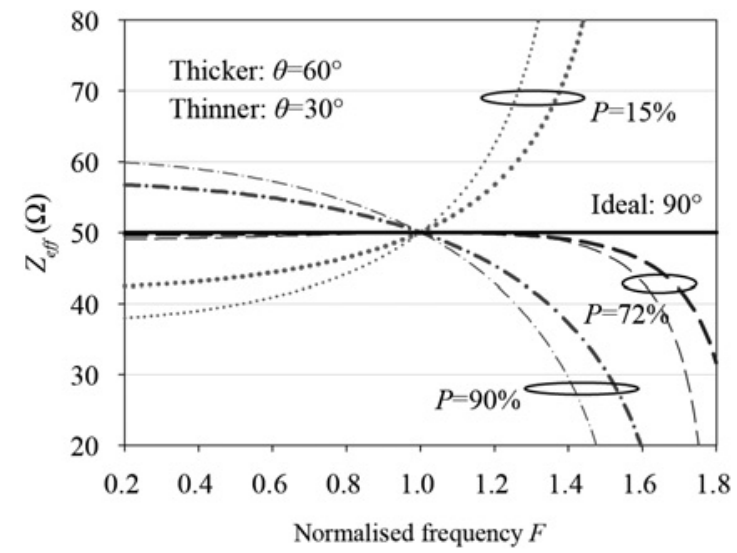

Fig. 4 Comparison of effective characteristic impedance: ideal $\left(90^{\circ}\right)$ with $Z_{0}=50 \Omega$ and miniaturised lines of $\theta=60^{\circ}$ (thicker lines) and $30^{\circ}$ (thinner lines) with two $(n=2)$ series inductors at $P=15,72$ and $90 \%$ 


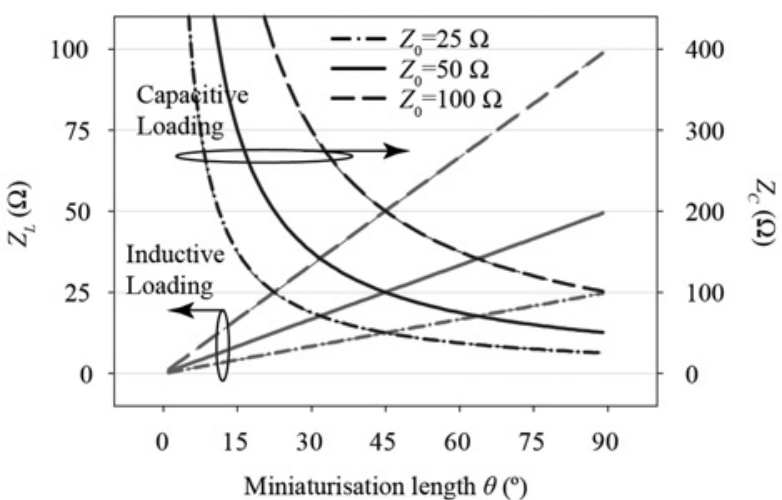

a

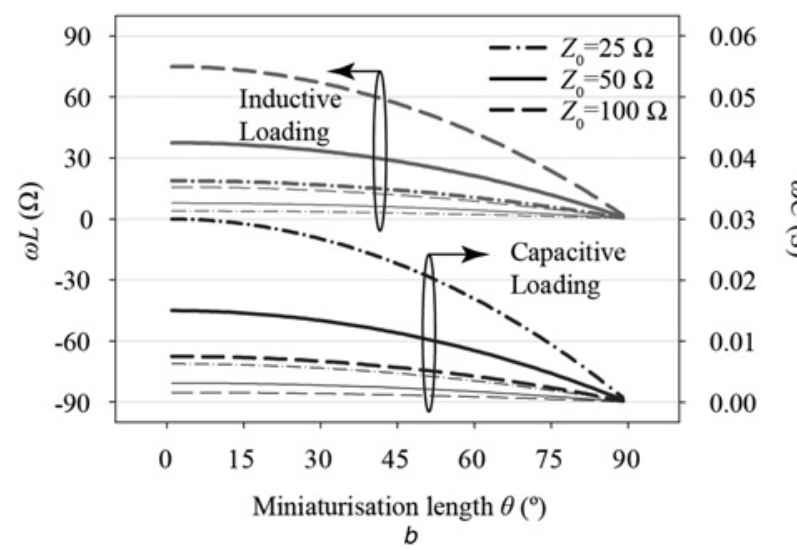

Fig. 5 Design curves for miniaturisation of quarter-wavelength lines with $n=2$ (thicker lines) and 10 (thinner lines) loaded at optimal locations $a$ Characteristic impedance

$b$ Reactance and susceptance levels

the line. However, this may not coincide with the location of equally spaced loading $(P=66.7 \%)$ [8], especially when $n$ is low. The optimum location maximises the physical resemblance between the unloaded and loaded lines for a given $n$ and $\theta$. Mathematically, the location minimises the $L_{2}$ norm of the difference in the $S$ parameter sets before and after miniaturisation

$$
\delta=\sqrt{\left|S_{11}^{\mathrm{C}}-S_{11}^{\mathrm{R}}\right|^{2}+\left|S_{21}^{\mathrm{C}}-S_{21}^{\mathrm{R}}\right|^{2}}
$$

where the superscripts $C$ and $R$ denote conventional and reactively loaded lines, respectively. As a result, performance degradation is minimised and can be improved somewhat further by increasing $n$ and/or $\theta$.

These results can be applied to conventional quarter-wavelength lines of any characteristic impedance. Therefore circuits that consist of various quarter-wavelength transmission lines (e.g. branch-line hybrid couplers, ring hybrid couplers, Wilkinson power dividers and quarter-wavelength impedance transformers) will maintain their performance nearly intact before and after miniaturisation.

Fig. 6 compares the ideal responses of single- and multi-section Chebyshev impedance transformers that match 25-50 $\Omega$. The conventional transformers in [1] are miniaturised by the previous loading methods in $[4,8]$, as well as by the proposed method. For example, the design parameters for a miniaturised impedance transformer based on $\theta=30^{\circ}$ sections in Fig. $6 a$ are $Z_{\mathrm{C}}=105.39 \Omega$ and $C=3.01 \mathrm{pF}$ for the proposed method, and $Z_{\mathrm{C}}=106.08 \Omega$ and $C=3.14 \mathrm{pF}$ for the method in [8]. The critical effect of loading location can be evidenced clearly by comparing the performances of the two transformers with nearly the same design parameters. This shows that the proposed method provides a remarkable improvement in performance that closely matches the response of the ideal circuit, especially for a higher miniaturisation rate.
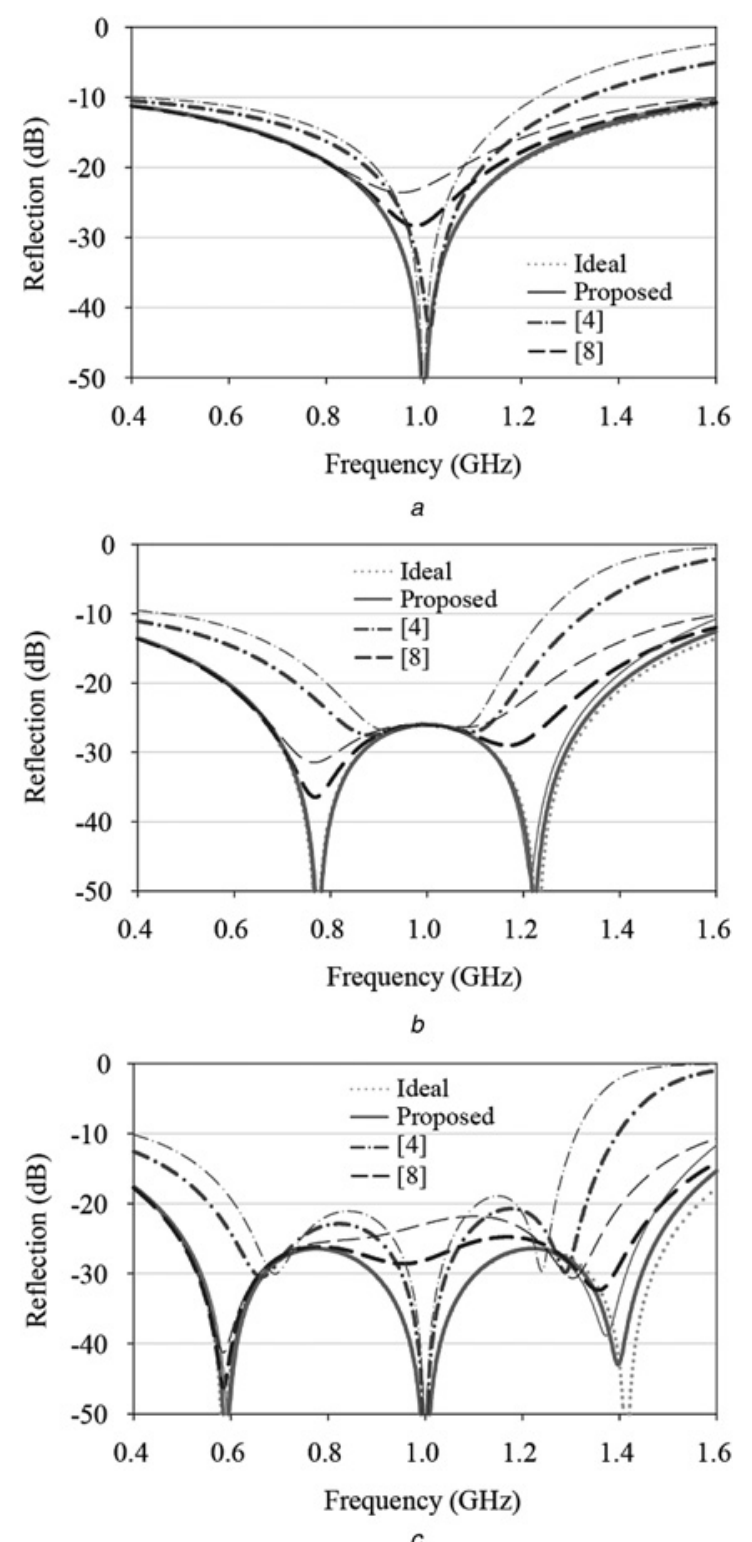

Fig. 6 Ideal circuit simulation results of Chebyshev impedance transformers with $\Gamma_{m}=0.05$ based on quarter-wavelength $\left(90^{\circ}\right)$ and miniaturised transmission lines of $\theta=30^{\circ}$ (thinner lines) and $60^{\circ}$ (thicker lines) with $n=2$

All impedance transformers match $25-50 \Omega$

$a$ Single-section

$b$ Two-section

$c$ Three-section

Furthermore, the effect of optimal loading is even more substantial as more sections are cascaded to achieve a wider bandwidth. As the number of cascaded sections increases, the response becomes even worst because performance degradation in each section accumulates. However, the proposed method provides a response that is much closer to the ideal response even when three sections are cascaded, as can be seen in Fig. 6c. Finally, although the optimal locations in Fig. 3 have been calculated for lossless cases, the effect of loss in practical transmission lines is minor. This will be validated by the experimental results in Section 4 .

\section{Experimental results of multi-section transformers}

For experimental verification, two transformers are designed at 1.5 $\mathrm{GHz}$ to have a Chebyshev response with $\Gamma_{m}=0.05$. Transformer A 
is a two-section transformer that matches $25-50 \Omega$ in a $66 \%$ bandwidth, whereas Transformer B is a three-section transformer that matches $100-50 \Omega$ in a larger bandwidth of $99 \%$.

The proposed method of reactive loading is capable of providing not only characteristics of an ideal miniaturisation in terms of performance, but also flexibility in design. For example, for Transformer A, each section is miniaturised to a different length so that all loading capacitances are the same. Then the transformer can be fabricated with identical surface-mount-device (SMD)-type capacitors, allowing for low-cost fabrication. For Transformer B, each section is miniaturised to a different length and a different $n$ so that all transmission line sections are of the same characteristic impedance. This is another way of simplifying the design process because all junction discontinuities exhibit nearly the same parasitic effects. In all cases, capacitors (or inductors) are loaded at the optimal locations in Fig. 3.

The design procedure for Transformer A is as follows. First, $\theta=$ $36^{\circ}$ with $n=2$ is chosen for the first section, which is originally of characteristic impedance $41 \Omega$. In this case, the lengths of transmission-line sections are $\theta_{1}=7.88^{\circ}$ and $\theta_{2}=20.24^{\circ}$ for the optimal $P$ of $72 \%$ in Fig. 3. Then, $1.63 \mathrm{pF}$ of shunt capacitance is calculated with $(5 \mathrm{~b})$ and the characteristic impedance of $Z_{C}=$ $102.40 \Omega$ with (5a). For the second section with a $30.5 \Omega$ impedance, $\theta=55.7^{\circ}$ with $n=2$ is chosen so that it can be miniaturised also with $1.63 \mathrm{pF}$, the same capacitance as the first section. Finally, $Z_{C}=49.2 \Omega$ is calculated with (5a) for the second section. Other transformers can be designed in a similar way. For $n=3$, the design parameters can be calculated by (11). For a higher $n$, they can be obtained numerically. If the parameters are impractical, $\theta$ and/or $n$, and/or the type of loading, can be changed. The design parameters for both transformers are

Table 1 Design parameters for Transformers A and B

\begin{tabular}{|c|c|c|c|c|}
\hline \multirow[b]{2}{*}{ Section } & \multicolumn{2}{|c|}{ Transformer A } & \multicolumn{2}{|c|}{ Transformer B } \\
\hline & Conventional & Miniaturised & Conventional & Miniaturised \\
\hline 1 & $\begin{array}{c}90^{\circ} \\
Z_{0}=41 \Omega\end{array}$ & $\begin{array}{c}\theta=36^{\circ}, n=2 \\
P=72 \% \\
Z_{C}=102.4 \Omega \\
C=1.63 \mathrm{pF}\end{array}$ & $\begin{array}{c}90^{\circ} \\
Z_{0}=57.4 \Omega\end{array}$ & $\begin{array}{c}\theta=46.5^{\circ}, n=3, \\
P=70.2 \% \\
Z_{L}=29.7 \Omega, \\
L=2.30 \mathrm{nH}\end{array}$ \\
\hline 2 & $\begin{array}{c}90^{\circ} \\
Z_{0}=30.5 \Omega\end{array}$ & $\begin{array}{c}\theta=55.7^{\circ}, n=2, \\
P=72 \% \\
Z_{C}=49.2 \Omega, \\
C=1.63 \mathrm{pF}\end{array}$ & $\begin{array}{c}90^{\circ} \\
Z_{0}=70.7 \Omega\end{array}$ & $\begin{array}{c}\theta=37.7^{\circ}, n=4, \\
P=69.4 \% \\
Z_{L}=29.7 \Omega, \\
L=2.41 \mathrm{nH}\end{array}$ \\
\hline 3 & - & - & $\begin{array}{c}90^{\circ} \\
Z_{0}=87.2 \Omega\end{array}$ & $\begin{array}{c}\theta=30.6^{\circ}, n=5, \\
P=68.9 \% \\
Z_{L}=29.7 \Omega, \\
L=2.55 \mathrm{nH}\end{array}$ \\
\hline
\end{tabular}

summarised in Table 1. All miniaturisation lengths in this paper are chosen for demonstration purposes. The miniaturisation rate can be increased further by increasing the reactance and/or the total number of loading $n$, yet performance degradation still can be minimised by loading at the locations provided in Fig. 3 .

For demonstration, Transformer A is miniaturised using SMD-type lumped elements, whereas Transformer B is miniaturised with series inductors in distributed forms of high-impedance $(123 \Omega)$ meandered lines. SMD-type lumped elements allow for compact size, but often suffer from tolerance in their values, parasitic effects and relatively high loss because of notorious low- $Q$ properties. On the other hand, the performance of distributed elements can be predicted more accurately, which simplifies the design procedure. However, they generally require a larger size.

The layout has been optimised with a full-wave simulator to compensate for the parasitic effects of lumped elements and junction discontinuities. Fig. 7 shows the detailed dimensions of the final layout of both transformers on an RF-35 substrate from Taconic with a relative permittivity of 3.5 , a thickness of $0.76 \mathrm{~mm}$ and a loss tangent of 0.0018. All transformers are realised in a two-port configuration. The characteristic impedance of the output feeding line is same as the impedance that the transformer is designed to match. For example, a $100 \Omega$ line is attached to the port 2 of Transformer B. However, a $50 \Omega$ line is attached to port 2 of Transformer A since a $25 \Omega$ line is too wide to fit in the 3680-20 test fixture in which the samples are placed during measurement. Shown in Fig. 8 is a photograph of all fabricated transformers, along with the corresponding conventional quarter-wavelength transformers. The size reduction factor calculated from the physical lengths is $(1-30.38 \mathrm{~mm} \div 59.13$ $\mathrm{mm}) \times 100=49 \%$ and $(1-42.01 \mathrm{~mm} \div 93.2 \mathrm{~mm}) \times 100=55 \%$ for Transformers $\mathrm{A}$ and $\mathrm{B}$, respectively. Owing to the non-zero lengths of semi-lumped elements, the size reduction factor is slightly smaller than the miniaturisation rate of $\left(1-114.8^{\circ} \div 270^{\circ}\right)=$ $57.5 \%$ for Transformer B.

Measurement is performed from 0.5 to $2.5 \mathrm{GHz}$ using an MS4624D vector network analyser from Anritsu. Line-reflect-line calibration sets the reference planes at the end of the feeding lines, immediately inside the test fixture. The reference planes of calibration are shown for Transformer B in Fig. 7. The effect of non-50 $\Omega$ ports can be calibrated out by simply assigning the intended impedance as the port impedance when the measured data are post-processed. For example, the $S$-parameters of Transformer B, which matches $50-100 \Omega$, can be obtained by post-processing the measured data with $100 \Omega$ as the port impedance of the output port. At the same time, the 10-mm-long feeding lines at both ends can also be calibrated out.

Fig. 9 shows the measured results. Provided in the same figure are full-wave simulation results by high-frequency structure simulator

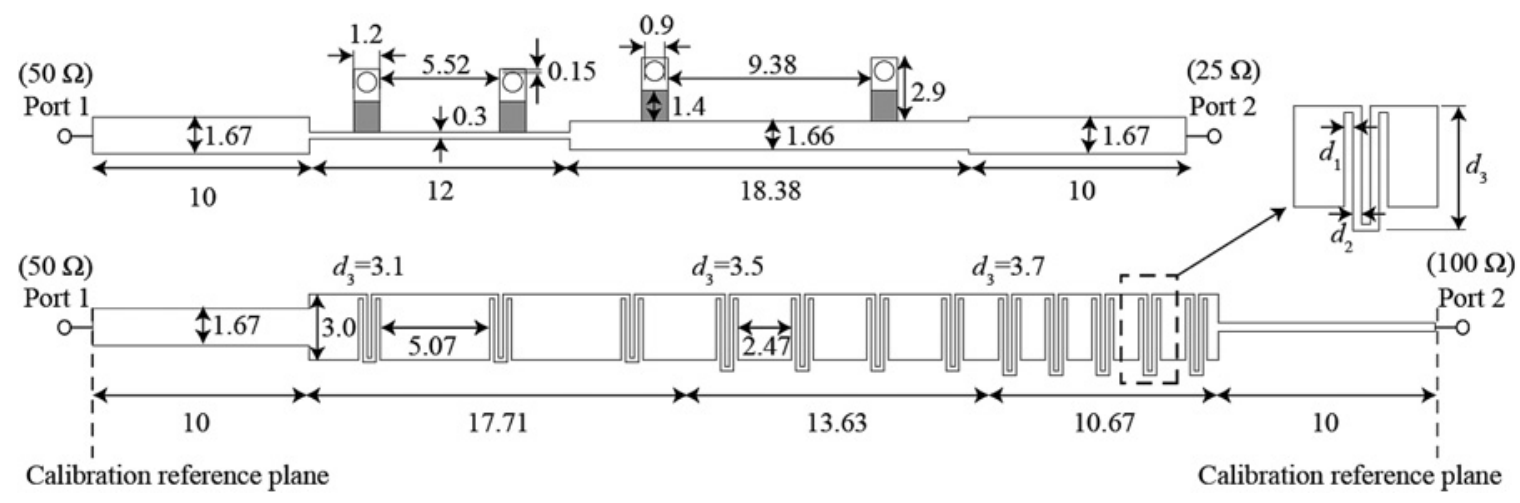

Fig. 7 Final layouts of Transformer A (top) and Transformer B (bottom)

For Transformer A, 2012-sized SMD-type capacitors are placed in shaded regions Circles indicate via-holes

For transformer B, $d_{1}=d_{2}=0.2 \mathrm{~mm}$ in all inductors

All dimensions are in millimetres 


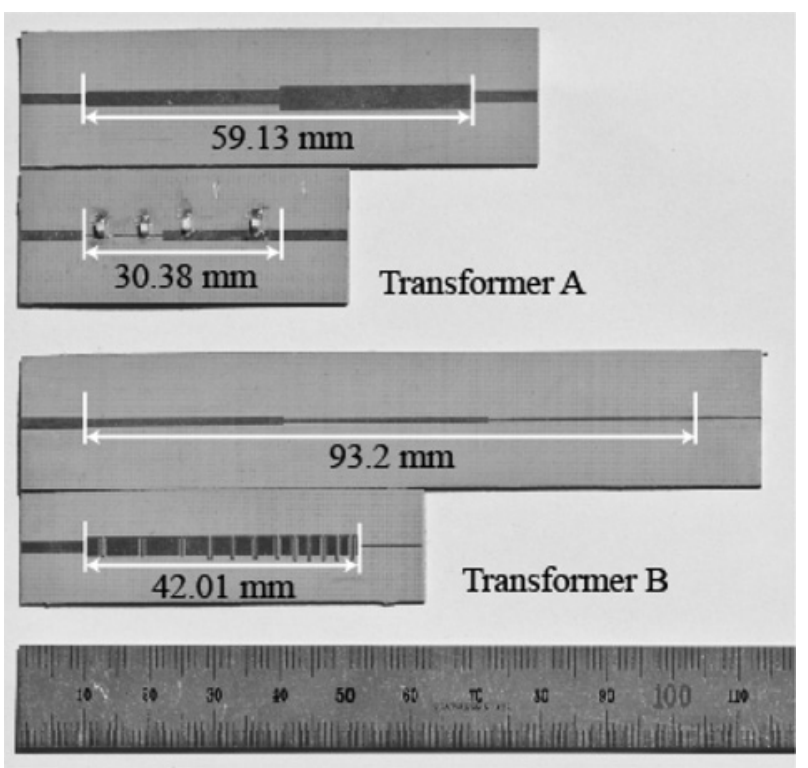

Fig. 8 Photograph of fabricated multi-section impedance transformers

[33] for comparison and ideal circuit simulation results for conventional transformers for reference. The measured reflection of both the two-section and three-section transformers shows strong agreement with the ideal responses. They also demonstrate
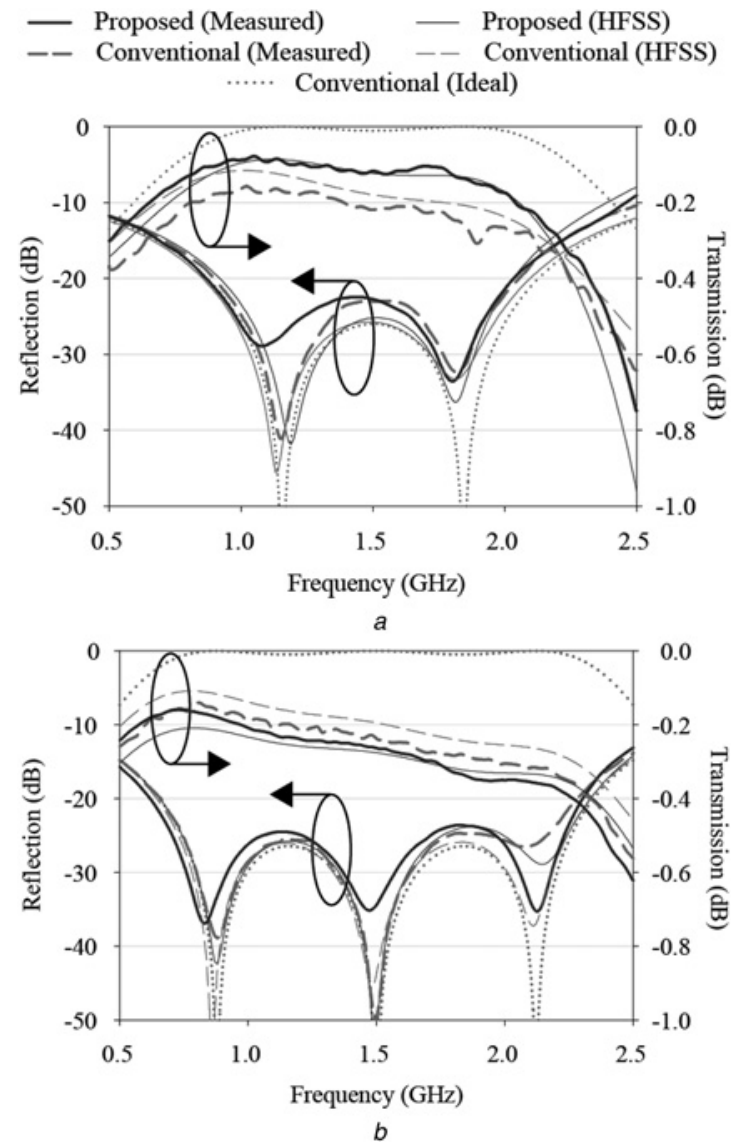

Fig. 9 Measured (thicker lines) and full-wave simulated (thinner lines) results of conventional and miniaturised multi-section Chebyshev impedance transformers with $\Gamma_{m}=0.05$

Ideal (dotted lines) responses are also given for reference

$a$ Two-section transformer that matches $25-50 \Omega$

$b$ Three-section transformers that matches $100-50 \Omega$ little deterioration from their corresponding conventional counterparts within the specified ripple bandwidths of 66 and $99 \%$, while maintaining nearly the same ripple level of $\Gamma_{m}=0.05$ or a reflection of $26 \mathrm{~dB}$ even with more than $55 \%$ reduction in size. When the reflection levels remain low within the passband, the insertion loss is dominated by the ohmic loss. This is governed by the skin effect, which is verified by the measured transmission that decreases as the frequency increases, showing good agreement with the simulated results. The results in Fig. 9 for various $n$ and various $\theta$ verify the effectiveness of the proposed approach. Finally, a comparison of measured transmissions shows that the effect of miniaturisation on loss performance is negligible, even in Transformer A, which is based on SMD lumped capacitors.

\section{Conclusion}

This paper demonstrated an improved miniaturisation method for quarter-wavelength transmission lines that provides minimal degradation in performance. The optimal location of reactive loading maintains responses before and after miniaturisation. Compared with previous methods of reactive loading, a remarkable improvement in performance is achieved in reactively loaded circuits, especially when a number of sections are cascaded for bandwidth enhancement. Moreover, the method provides design flexibility that allows lines to be miniaturised with various conditions and still maintain minimal performance degradation. Furthermore, the freedom in choosing $n, \theta$ and the type of loading allows the proposed technique to be applied to $\lambda / 4$ lines of all impedances, and the method can also be extended to miniaturise transmission lines of any lengths. Further investigation of the effects of transmission-line sections with incommensurate lengths remains as future work, and as a possible solution to relieve the practical limitations of the proposed method of miniaturisation.

\section{Acknowledgments}

This work was partly supported by the ICT R\&D program of MSIP/ IITP [12-911-01-108, Massive MIMO technology based on space-polarization division multiple access for super-macro cellular system] and by the National Research Foundation of Korea (NRF) grant funded by the Korea government (MEST) (no. 2011-0016802).

\section{References}

Pozar, D.M.: 'Microwave engineering' (John Wiley \& Sons, Inc., Hoboken, NJ, 2005, 3rd edn.)

2 Hirota, T., Minakawa, A., Muraguchi, M.: 'Reduced-size branch-line and rat-race hybrids for uniplanar MMIC's', IEEE Trans. Microw. Theory Tech., 1990, 38, (3), pp. $270-275$

3 Hsu, C.-L., Kuo, J.-T., Chang, C.-W.: 'Miniaturized dual-band hybrid couplers with arbitrary power division ratios', IEEE Trans. Microw. Theory Tech., 2009, 57, (1), pp. 149-156

4 Hettak, K., Morin, G.A., Stubbs, M.G.: 'The integration of thin-film microstrip and coplanar technologies for reduced-size MMICs', IEEE Trans. Microw. Theory Tech., 2005, 53, (1), pp. 283-291

5 Park, M.-J.: 'Dual-band, unequal length branch-line coupler with center-tapped stubs', IEEE Microw. Wirel. Compon. Lett., 2009, 19, (10), pp. 617-619

6 Scardelletti, M.C., Ponchak, G.E., Weller, T.M.: 'Miniaturized Wilkinson power dividers utilizing capacitive loading', IEEE Microw. Wirel. Compon. Lett., 2002, 12, (1), pp. 6-8

7 Lee, S., Lee, Y.: 'Generalized miniaturization method for coupled-line bandpass filters by reactive loading', IEEE Trans. Microw. Theory Tech., 2010, 58, (9), pp. 2383-2391

8 Rawat, K., Ghannouchi, F.M.: 'A design methodology for miniaturized power dividers using periodically loaded slow wave structure with dual-band applications', IEEE Trans. Microw. Theory Tech., 2009, 57, (12), pp. 3380-3388

9 Issa, H., Duchamp, J.-M., Abou-Chahine, S., Ferrari, P.: 'Loaded miniature transmission lines compatible with low cost substrates: $Q$-factor improvement'. 40th European Microwave Conf. Digest, 2010, pp. 1218-1221

10 Chuang, M.-L.: 'Miniaturized ring coupler of arbitrary reduced size', IEEE Microw. Wirel. Compon. Lett., 2005, 15, (1), pp. 16-18

11 Eccleston, K.W., Ong, S.H.M.: 'Compact planar microstripline branch-line and rat-race couplers', IEEE Trans. Microw. Theory Tech., 2003, 51, (10), pp. 2119-2125 
12 Ghali, H., Moselhy, T.A.: 'Miniaturized fractal rat-race, branch-line, and coupled-line hybrids', IEEE Trans. Microw. Theory Tech., 2004, 52, (11), pp. $2513-2520$

13 Sun, K.-O., Ho, S.-J., Yen, C.-C., van der Weide, D.: 'A compact branch-line coupler using discontinuous microstrip lines', IEEE Microw. Wirel. Compon. Lett., 2005, 15, (8), pp. 519-520

14 Wang, C.-W., Ma, T.-G., Yang, C.-F.: 'Miniaturized branch-line coupler with harmonic suppression for RFID applications using artificial transmission lines'. IEEE MTT-S Int. Microwave Symp. Digest, 2007, pp. 29-32

15 Tang, C.-W., Chen, M.-G., Tsai, C.-H.: 'Miniaturization of microstrip branch-line coupler with dual transmission lines', IEEE Microw. Wirel. Compon. Lett., 2008, 18, (3), pp. 185-187

16 Tseng, C.-H., Wu, C.-H.: 'Design of compact branch-line couplers using $\pi$-equivalent artificial transmission lines', IET Microw. Antennas Propag., 2012, 6, (9), pp. 969-974

17 Chun, Y.-H., Hong, J.-S.: 'Compact wide-band branch-line hybrids', IEEE Trans. Microw. Theory Tech., 2006, 54, (2), pp. 704-709

18 Tang, C.-W., Chen, M.-G.: 'Synthesizing microstrip branch-line couplers with predetermined compact size and bandwidth', IEEE Trans. Microw. Theory Tech., 2007, 55, (9), pp. 1926-1934

19 Jung, S.-C., Negra, R., Ghannouchi, F.M.: 'A design methodology for miniaturized $3 \mathrm{~dB}$ branch-line hybrid couplers using distributed capacitors printed in the inner area', IEEE Trans. Microw. Theory Tech., 2008, 56, (12), pp. 2950-2953

20 Xu, H.-X., Wang, G.M., Zhang, C.-X., Liang, J.-G.: 'Novel design of compact microstrip diplexer based on fractal-shaped composite right/left handed transmission line', J. Infrared Millim. Waves, 2011, 30, (5), pp. 390-457

$21 \mathrm{Xu}$, H.-X., Wang, G.M., Zhang, X.-K., Yang, X.-L.: 'Novel compact dual-band rat-race coupler combining fractal geometry and CRLH TLs', Wirel. Pers Commun., 2012, 66, (4), pp. 855-864

22 Xu, H.-X., Wang, G.-M., Zhang, C.-X., Yu, Z.-W., Chen, X.: 'Composite right/ left-handed transmission line based on complementary single-split ring resonator pair and compact power dividers application using fractal geometry', IET Microw. Antennas Propag., 2012, 6, (9), pp. 1017-1025
23 Xu, H.-X., Wang, G.M., Chen, X., Li, T.-P.: 'Broadband balun using fully artificial fractal-shaped composite right/left handed transmission line', IEEE Microw. Wirel. Compon. Lett., 2012, 22, (1), pp. 16-18

24 Sung, Y.J., Ahn, C.S., Kim, Y.-S.: 'Size reduction and harmonic suppression of rat-race hybrid coupler using defected ground structure', IEEE Microw. Wirel. Compon. Lett., 2004, 14, (1), pp. 7-9

25 García-García, J., Bonache, J., Martín, F.: 'Application of electromagnetic bandgaps to the design of ultra-wide bandpass filters with good out-of-band performance', IEEE Trans. Microw. Theory Tech., 2006, 54, (12), pp. 4136-4140

26 Lim, T.B., Sun, S., Zhu, L.: 'Compact ultra-wideband bandpass filter using harmonic-suppressed multiple-mode resonator', Electron. Lett., 2007, 43, (22), pp. 1205-1206

27 Sor, J., Qian, Y., Itoh, T.: 'Miniature low loss CPW periodic structures for filter applications', IEEE Trans. Microw. Theory Tech., 2001, 49, (12), pp. 2336-2341

28 Martín, F., Falcone, F., Bonache, J., Laso, M., Lopetegi, T., Sorolla, M. 'New CPW low pass filter based on a slow wave structure', Microw. Opt. Technol. Lett., 2003, 38, (3), pp. 190-193

29 Wang, C.-J., Lin, C.-H.: 'Compact lowpass filter with sharp transition knee by utilising a quasi- $\pi$-slot resonator and open stubs', IET Microw. Antennas Propag., 2010, 4, (4), pp. 512-517

30 Chen, C.-C., Tzuang, C.-K.C.: 'Synthetic quasi-TEM meandered transmission lines for compacted microwave integrated circuits', IEEE Trans. Microw. Theory Tech., 2004, 52, (6), pp. 1637-1647

31 Yun, Y.: 'A novel microstrip-line structure employing a periodically perforated ground metal and its application to highly miniaturized and low-impedance passive components fabricated on GaAs MMIC', IEEE Trans. Microw. Theory Tech., 2005, 53, (6), pp. 1951-1959

32 Koochakzadeh, M., Abbaspour-Tamijani, A.: 'Miniaturized transmission lines based on hybrid lattice-ladder topology', IEEE Trans. Microw. Theory Tech. 2010, 58, (4), pp. 949-955

33 'High Frequency Structure Simulator v10.0', Ansoft Corporation, Pittsburgh, PA, 2005 